\title{
Clinical Outcomes of Low-Cost, Anchorless Repair of the Triceps Tendon Using a Proximal Knot Technique
}

\author{
Robert R. Hall III, B.S., Alison K. Sarokhan, M.D., and Nicky L. Leung, M.D.
}

Purpose: To use validated outcome measures to evaluate the clinical results of surgical repair of distal triceps tendon ruptures using transosseous tunnels and high-strength sutures with proximally based knots. Methods: A consecutive series of traumatic distal triceps tendon ruptures at a single institution was studied. All cases were surgically repaired by 1 surgeon using high-strength suture with a bone tunnel-based repair technique. Repair knots were oriented proximally instead of in the traditional distal position. All patients were evaluated at long-term follow-up with a physical examination performed by the orthopaedic surgeon and the following validated outcome measures: Disabilities of the Arm, Shoulder and Hand score; Mayo Elbow Performance Score; and visual analog scale score. Results: Seven male patients with a mean age of 38 years (range, 19-50 years) and mean follow-up period of $4.1 \pm 1.2$ years underwent distal triceps tendon repair with bone tunnels and high-strength sutures with proximally positioned knots. Of the repairs, 4 involved the dominant arm. At final follow-up, the mean Disabilities of the Arm, Shoulder and Hand score was $1.3 \pm 3.1$; the mean Mayo Elbow Performance Score was 99.3 \pm 1.9; and the mean visual analog scale score was 0. One additional patient who declined participation in the study had wound dehiscence and infection with an associated partial rerupture. Conclusions: This case series of triceps tendon repairs using transosseous tunnels and proximally based knots showed favorable postoperative elbow function based on validated outcome measures. Level of Evidence: Level IV, therapeutic case series.

$\mathbf{T}$ he triceps brachii is a 3-headed muscle whose tendon attaches at the olecranon process ${ }^{1}$ and functions to enable extension of the elbow. Distal triceps ruptures are a relatively rare entity ${ }^{2}$ and usually occur in athletes, weightlifters, or anabolic steroid users. $^{3,4}$ Trauma, especially a laceration or fall on an outstretched hand, has been implicated in acute triceps tendon rupture. ${ }^{5}$ Although rare, such injuries are accompanied by significant impairment in upper-limb function. $^{6,7}$ As such, surgical repair of complete

From Tufts University School of Medicine, Boston, Massachusetts, U.S.A. (R.R.H.); Department of Orthopaedic Surgery, Tufts Medical Center, Boston, Massachusetts, U.S.A. (A.K.S.); and Department of Orthopaedic Surgery, Newton-Wellesley Hospital, Newton, Massachusetts, U.S.A. (N.L.L.).

The authors report no conflicts of interest in the authorship and publication of this article. Full ICMJE author disclosure forms are available for this article online, as supplementary material.

Received August 12, 2020; accepted December 9, 2020.

Address correspondence to Robert R. Hall III, B.S., Tufts University School of Medicine, 145 Harrison Ave, Boston, MA 02111,U.S.A.E-mail:rrhall95@ gmail.com

(C) 2021 THE AUTHORS. Published by Elsevier Inc. on behalf of the Arthroscopy Association of North America. This is an open access article under the CC BY-NC-ND license (http://creativecommons.org/licenses/by-nc-nd/4.0/). 2666-061X/201381

https://doi.org/10.1016/j.asmr.2020.12.005 triceps tendon ruptures is recommended for all healthy individuals. $^{8}$

Traditional transosseous techniques for triceps repair have required tying large knots distally on the subcutaneous surface of the proximal ulna, a potential source of postoperative pain. ${ }^{9,10}$ Although knotless suture anchor repair eliminates the necessity for such knots, ${ }^{11,12}$ it is associated with an increase in equipment cost.

Recent literature has described a transosseous repair with proximal knots tied over the triceps tendon, with the goal of minimizing the potential for symptomatic prominent suture knots without the added cost of anchors. ${ }^{13}$ Although favorable results have been published for numerous other methods, ${ }^{6,14}$ a clinically superior technique has yet to be proved. The purpose of our study was to use validated outcome measures to evaluate the clinical results of surgical repair of distal triceps tendon ruptures using transosseous tunnels and high-strength sutures with proximally based knots. Our hypothesis was that this technique would achieve adequate restoration of elbow extensor strength and patient satisfaction while minimizing the potential for symptomatic prominent suture knots and without the additional cost of anchors. 


\section{Methods}

After receiving institutional review board approval (Partners Healthcare protocol No. 2019P000464), we performed a retrospective chart review to identify patients who underwent triceps tendon repair surgery from January 2011 to January 2019 performed by a single surgeon (N.L.L.) at 1 institution. All patients aged between 18 and 85 years who experienced isolated, acute, insertional triceps tears fixed with bone tunnels and proximal knots as described by Sarokhan and Leung $^{13}$ were included. The exclusion criteria included chronic injury, enthesophyte removal, ipsilateral biceps injury, and myotendinous rupture. All patients experienced partial or complete triceps tears as diagnosed by physical examination findings and magnetic resonance imaging or radiography showing a proximally retracted avulsion fragment. Patients underwent primary repair surgery for discomfort and objective weakness.

The primary diagnosis, demographic characteristics, mechanism of injury, procedure, date of surgery, and injury-related information were obtained from the electronic medical record. Patients were subsequently contacted by phone to follow up with the surgeon for a physical examination and to complete a series of questionnaires. Participants were evaluated with validated outcome measures including the Disabilities of the Arm, Shoulder and Hand (DASH) score; the DASH Work and Sports/Arts optional sections; the Mayo Elbow Performance Score (MEPS); and the visual analog scale (VAS) score. Patients were also evaluated with a Likert satisfaction score and a focused physical examination that included strength and range-ofmotion assessment.

\section{Surgical Technique}

The surgical technique as described by Sarokhan and Leung ${ }^{13}$ was used. The patient was placed in the supine position after administration of regional anesthesia. Prior to sterile preparation of the surgical site, a nonsterile pneumatic tourniquet was applied to the arm as far proximal as possible.

To expose the surgical site, a $10-\mathrm{cm}$ longitudinal curvilinear incision was made, curving just lateral to the tip of the olecranon. The tendon stump was then identified and freed from surrounding tissues. Longitudinal incision of any intact paratenon was performed to allow preservation for later repair. The tendon end was grasped with a non-penetrating clamp over a damp sponge, with care taken to avoid damaging the ruptured end of the tendon. To allow further mobilization of the tendon, a small extension of the longitudinal split between the heads of the triceps was performed when necessary. Longitudinal tension was applied to the tendon stump for several minutes to lengthen the triceps muscle as much as possible. Boneto-tendon contact between the tendon stump and the

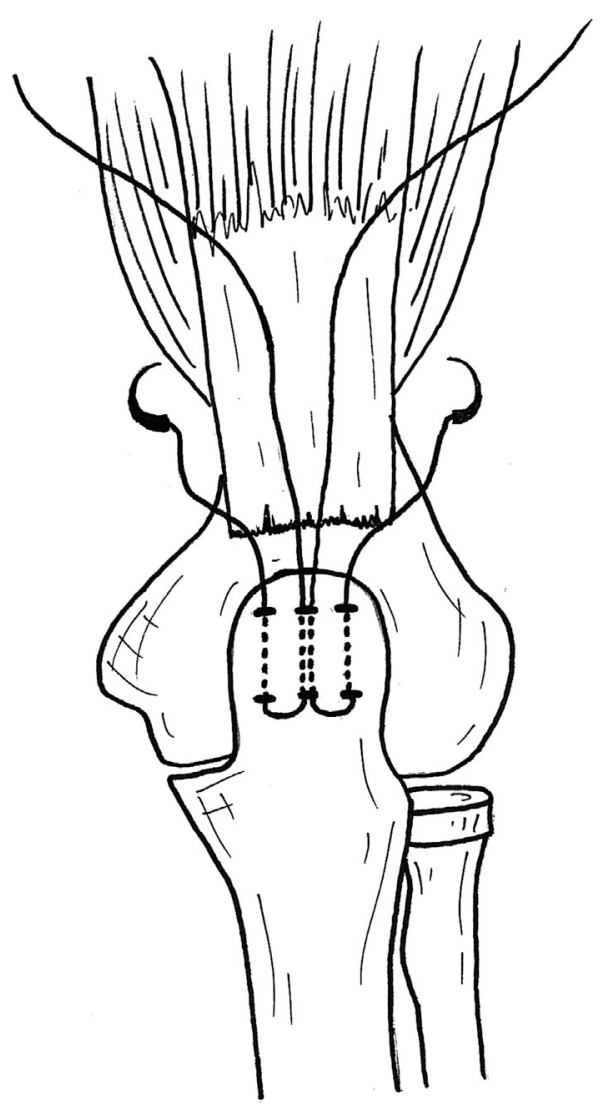

Fig 1. The suture ends with the needles should be exiting laterally and medially, with the free limbs exiting the central tunnel. Reprinted with permission from Sarokhan and Leung. ${ }^{13}$

native insertion of the olecranon process was facilitated by maintaining the elbow in slight extension. Residual tissue and debris were cleared from the bony footprint and a bleeding bed was created using a curette to promote healing.

Three holes were created in the central, radial, and ulnar aspects of the tendon footprint using a $2-\mathrm{mm}$ drill. Three additional holes were drilled through the dorsal cortex about $1 \mathrm{~cm}$ distal to the first 3 holes. The holes were drilled proximally and then distally and were connected using the large-curvature needle from a No. 5 Ethibond Excel suture (Ethicon, Somerville, $\mathrm{NJ}$ ), producing 3 curved bone tunnels. A nonabsorbable high-strength suture (No. 5 Ethibond Excel) was passed antegrade through the central tunnel and then shuttled retrograde through the most lateral tunnel. While the first suture was held taut, a second suture was passed antegrade through the central tunnel. To ensure that the second suture needle did not transect the first suture, the lateral suture was pulled back and forth and then passed retrograde through the most medial tunnel.

After suture passage, both suture needles exited the lateral and medial tunnels with the free limbs entering the central tunnel, as shown in Figure 1. A modified 


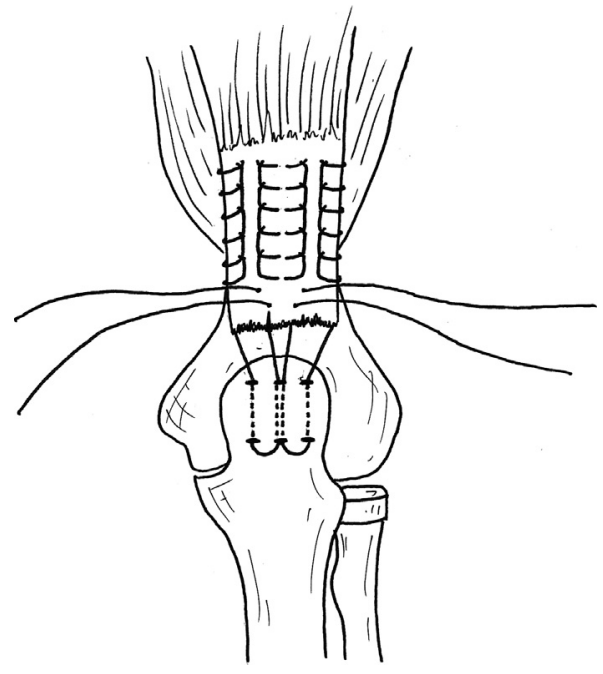

Fig 2. All sutures exit the dorsal surface of the tendon. Reprinted with permission from Sarokhan and Leung. ${ }^{13}$

Krackow locking-loop stitch was used to weave the lateral suture through the lateral half of the tendon. Next, the free end of the same suture was passed from deep to superficial within the tendon using a free needle. Both the post limb and the limb that was run proximally and distally in the tendon exited the tendon on the dorsal surface. The procedure was then repeated on the medial half of the tendon, which was also shuttled through the tendon using a modified Krackow locking-loop stitch, as depicted in Figure 2.

After all of the sutures were passed, 2 half-hitches were tied to allow the suture to bring the tendon to bone; then, 5 more reversed alternating half-hitches were tied over the post limb with the elbow maintained in extension. The knots, tied in this manner, wound up on the dorsal surface of the triceps tendon about $1 \mathrm{~cm}$ proximal to the olecranon tip, where they were covered by a layer of fat at the time of skin closure. The sutures were then cut, and the elbow was flexed to $90^{\circ}$ to ensure that there was no gapping between the tendon and bone. If gapping occurred prior to reaching $90^{\circ}$ of flexion, the elbow was cast at the position of flexion that did not produce gapping.

A nonabsorbable suture was used to repair the longitudinal split between the heads of the triceps if present. Any intact overlying paratenon was repaired using a fine absorbable suture. The skin was closed in a layered manner, and a long arm cast was applied to immobilize the elbow at the aforementioned position of flexion that prevented repair gapping.

The postoperative protocol was similar for all patients. The elbow remained in a cast until 2 weeks postoperatively, at which time the sutures were removed and the elbow was recast at $90^{\circ}$ of flexion. At 4 weeks, the cast was removed. Patients were given a sling and started physical therapy allowing full active and passive range of motion at the time of cast removal. No resisted active extension of the elbow was allowed at initial cast removal. At 8 weeks, gentle resisted extension was initiated. At 12 weeks, patients were allowed unrestricted activity.

\section{Results}

During the study period, 17 triceps repair cases were performed by the senior surgeon. Each case was screened and evaluated for inclusion in this study. We excluded 1 patient who was aged younger than 18 years, 3 patients who underwent surgery for chronic tendinitis with enthesophyte excision to remove the calcified tendon area and then reattach the tendon, 1 patient who underwent a myotendinous repair technique with suture only, and 1 patient who had a nonconcomitant ipsilateral distal biceps injury. A total of 11 insertional ruptures were identified to be included in the analysis of patients who underwent repair with bone tunnels and proximal knots. These 11 patients were contacted by phone to participate in the study, which involved a physical examination by the surgeon and a series of subjective questionnaires. Of the 11 remaining patients, 3 were lost to follow-up and 1 declined to participate. The latter patient had experienced a postoperative infection and subsequent partial rerupture. This left a total of 7 patients for inclusion in the final analyses.

Patient demographic characteristics are presented in Table 1. All patients were men, with a mean age of 38 years (range, 19-50 years; standard deviation [SD], 13.3 years). Of the injuries, 5 occurred after falls during participation in athletics, 1 occurred during a fall from a height, and 1 occurred after a patient struck his arm on a door frame. Participants were largely free of comorbidities because none had a history of anabolic steroid use or kidney disease. One patient was an occasional smoker. Of the surgical repairs in these patients, 4 were performed on the dominant arm. Three repairs were performed for complete tears, whereas 4 repairs were performed for high-grade partial tears significant enough to cause notable weakness and ongoing pain.

The time from injury to surgery, time to follow-up, and range of motion and strength at follow-up are presented in Table 2. After acute tears, operative repair was performed in all patients with a mean time to surgery of 54.4 days (range, 10-105 days; SD, 39.5 days). The mean time to follow-up was 4.1 years (range, 2.4-5.3 years; SD, 1.2 years). Range of motion showed an arc from $0^{\circ}$ to $140^{\circ}$ of flexion with full supination and pronation after physical examination in all 7 patients. Grade 5 strength of elbow extension on physical examination was regained in each respondent. No complications or areas of irritation related to prominent knot stacks were found. These knot stacks could be identified on physical examination, but no 
Table 1. Demographic Data

\begin{tabular}{|c|c|c|c|c|c|c|c|}
\hline Patient No. & Age, yr & Sex & $\begin{array}{c}\text { Dominant vs } \\
\text { Nondominant Arm }\end{array}$ & $\begin{array}{l}\text { Mechanism of } \\
\text { Injury }\end{array}$ & Medical History & Smoker & $\begin{array}{c}\text { Partial vs } \\
\text { Complete in OR }\end{array}$ \\
\hline $\bar{l}$ & 46 & $M$ & Dominant & Fall from height & Healthy & No & Complete \\
\hline 2 & 19 & M & Dominant & $\begin{array}{l}\text { Fall during } \\
\text { collegiate bike } \\
\text { race }\end{array}$ & Healthy & No & Partial $(90 \%)$ \\
\hline 3 & 42 & M & Dominant & Fall off alpine slide & Kidney stones and psoriasis & No & Partial $(80 \%)$ \\
\hline 4 & 50 & M & Nondominant & Fall during hockey & Asthma & No & Partial $(60 \%)$ \\
\hline 5 & 50 & M & Dominant & Fall during lacrosse & Arthritis & No & Complete \\
\hline 6 & 40 & M & Nondominant & $\begin{array}{l}\text { Arm struck against } \\
\text { door frame }\end{array}$ & Depression & No & Partial $(60 \%)$ \\
\hline 7 & 20 & M & Nondominant & Fall during rugby & Healthy & Some days & Complete \\
\hline
\end{tabular}

M, male; OR, operating room.

patient noticed their presence or requested knot-stack removal.

Outcome measures, occupation, and sports are presented in Table 3. Further evaluation comprised the DASH score, DASH Work score, DASH Sports/Arts score, MEPS, VAS score, and postoperative patient satisfaction reporting (1-5). The average DASH score was 1.3 (range, $0-8.3$; SD, 3.1), indicating excellent outcomes in all participants. The mean MEPS was 99.3 (range, 95-100; SD, 1.89). Regarding the MEPS, only 1 patient reported a score of 95, with all other respondents reporting a score of 100. The same patient also reported a satisfaction score of 4 of 5 because he experienced occasional ulnar nerve symptoms during elbow flexion. All other patients reported a satisfaction score of 5 of 5 . The mean satisfaction score was 4.86 (range, 4-5; SD, 0.38). All patients reported a VAS pain score of 0 . Each of the 7 respondents was able to return to work without restriction. Each reported a DASH Work score of 0. Five patients indicated participation in sports, with 1 participating in golf alone, 1 participating in competitive road cycling, 1 participating in lacrosse and soccer, and 2 participating in multiple sports, as detailed in Table 3. Two patients indicated no participation in sports. Of note, the only suboptimal DASH Sports/Arts score was 75, which was reported in the high-level cyclist.

\section{Discussion}

Our study used standardized outcome measures and physical examination findings to show favorable outcomes in patients who underwent a previously described transosseous triceps repair technique. This method aimed to simplify the repair, minimize cost, and use proximal knots, potentially reducing painful knot prominence on the dorsal forearm. Distal triceps injuries are a relatively uncommon entity and usually occur in male individuals during athletics ${ }^{15}$ or after falls on outstretched arms, ${ }^{4}$ as was indicated in our series. Traditional techniques for distal triceps repair use either heavy nonabsorbable sutures ${ }^{9}$ passed through bone tunnels or suture anchors ${ }^{16}$ to reattach the tendon to the olecranon process.

On the basis of this case review, we believe the described repair technique shows favorable long-term outcomes. No patients experienced complications or reoperation related to prominent knot stacks, and none requested knot-stack removal. One patient experienced an infection-related partial rerupture. This patient was a cigarette smoker, which has been shown to contribute to delayed wound healing ${ }^{17}$ and higher rates of postoperative surgical-site infection. ${ }^{18}$ Another complication was intermittent ulnar neuropathy, occurring in the competitive cyclist, limiting his ability to participate

Table 2. Time From Injury to Surgery, Time to Follow-up, and Range of Motion and Strength at Follow-up

\begin{tabular}{|c|c|c|c|c|c|}
\hline Patient No. & Time from Injury to Surgery, $\mathrm{d}$ & Time to Follow-up, yr & \multicolumn{2}{|c|}{ Range of Motion, ${ }^{\circ}$} & Strength $(0-5)$ \\
\hline$\overline{1}$ & 30 & 5.4 & $5-135 / 0-135$ & $90 / 90$ & 5 \\
\hline 3 & 10 & 4.6 & $5-135 / 5-130$ & $90 / 90$ & 5 \\
\hline 4 & 90 & 4.1 & $0-135 / 0-135$ & $90 / 90$ & 5 \\
\hline 5 & 10 & 2.6 & $5-135 /<5$ to 135 & $90 / 90$ & 5 \\
\hline
\end{tabular}


Table 3. Outcome Measures

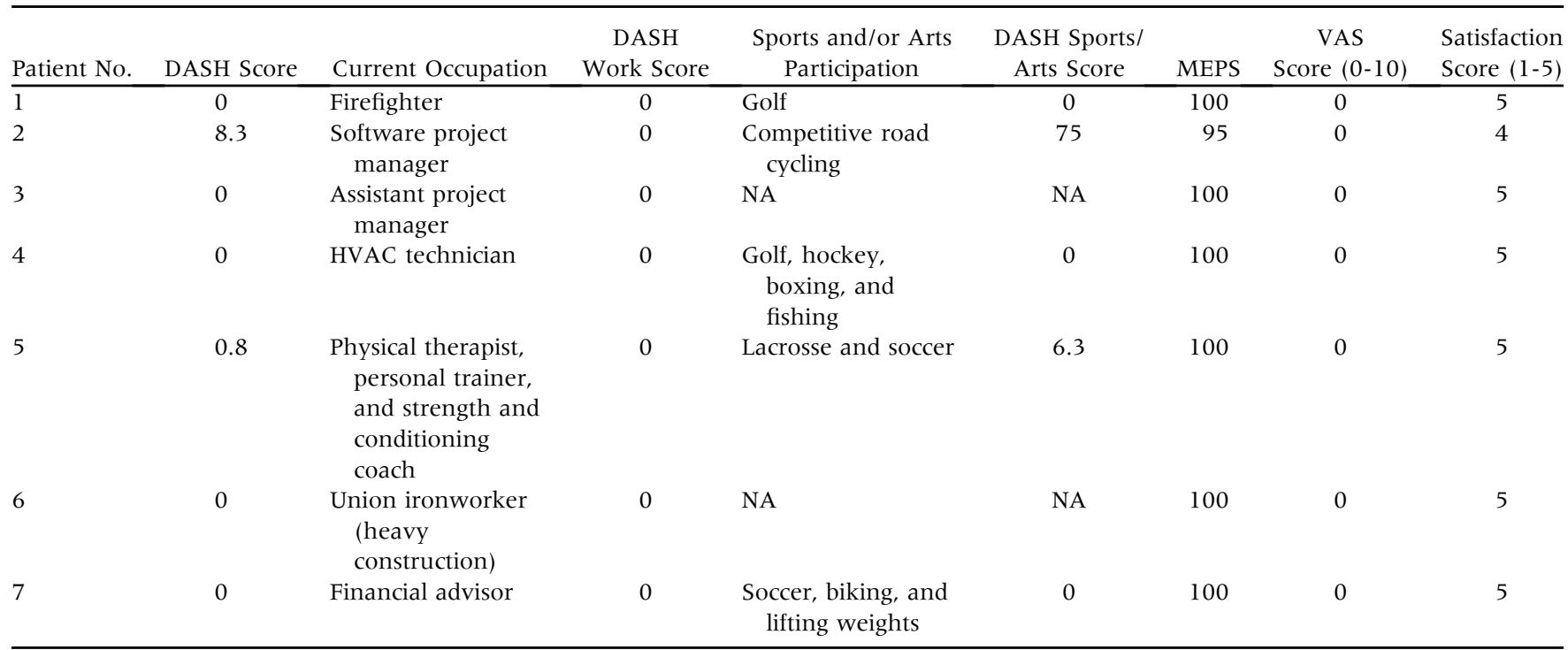

DASH, Disabilities of the Arm, Shoulder and Hand; HVAC, heating, ventilation, and air conditioning; MEPS, Mayo Elbow Performance Score; NA, not applicable; VAS, visual analog scale.

in this activity postoperatively. However, he declined further intervention for treatment of ulnar neuropathy.

We believe that this is the first triceps repair series that includes DASH sports-specific information. The inclusion of this evaluation tool may allow identification of more hardships than the general DASH instrument, especially in higher-level athletes. Detection of such limitations is especially critical in patients with triceps injuries because the demographic that experiences such injuries often participates in competitive or high-level athletics or activities. ${ }^{19}$

Mair et al. ${ }^{20}$ examined triceps injuries in National Football League (NFL) players during a 6-year span from 1991-1996. They included 10 partial and 11 complete triceps ruptures. Of the partial ruptures, 4 were surgically repaired, and all 10 patients continued playing in the NFL for at least 1 season after injury. Among the 11 complete ruptures that were surgically repaired, there was 1 rerupture; 1 player retired, whereas 10 played at least 1 season after injury. This finding shows the ability of athletes to return to a high level of performance after both complete and partial triceps tendon repairs. However, the article did not state which repair technique was used in its participants, limiting comparison or determination of an optimal technique in the competitive athlete.

In a subsequent case series, Finstein et al. ${ }^{21}$ examined 37 triceps repairs in NFL players from 20002009. Although all players returned to NFL play postoperatively, significant recovery times were reported, with players missing an average of 165 days (range, 49-318 days). Again, no information was provided on which type of repair was used in these patients, but it was shown that surgical repair allows patients to return to play. Of note, our case series included 5 cases of triceps repair in patients who participated in athletics, and all but 1 returned to play without complication, suggesting the efficacy of this technique in athletes.

Similarly, evaluations of military personnel have shown a return to high activity levels after triceps repair. A 2016 study of triceps repairs in 48 active-duty American military personnel showed that strength and function are adequately restored after surgery even in high-demand individuals. ${ }^{8}$ At a mean 26-month follow-up, 45 patients (94\%) had returned to active duty, $6(12.5 \%)$ experienced traumatic rerupture, and $4(8.3 \%)$ continued to experience chronic pain or weakness. Similar results were reported in a 2019 study of 37 repairs, in which $84 \%$ of participants returned to full military duty at 2 years. ${ }^{22}$ In this report, 1 participant experienced rerupture. A large group continued to experience discomfort, with $45 \%$ of patients reporting occasional elbow pain. Follow-up phone interviews with 14 members of the original cohort were conducted at an average of 52.4 months (range, 7-84.9 months). Participants reported an average DASH score of 4.7 (range, 0-15.9; SD, 4.7) and average MEPS of 85.4 (range, 60-100; SD, 11.7). Of these 14 patients, 12 $(85.7 \%)$ were satisfied. Our cases showed comparable outcomes to these studies of military personnel, specifically a lower average DASH score, a higher average MEPS, and high satisfaction rates. Although technique superiority cannot be concluded based on these comparisons, these data suggest that the outcomes found in our cases were similar to those in other series examining outcomes that included individuals who participated in high levels of activity. 
Further studies have shown good outcomes in general populations for both the transosseous technique and the suture anchor technique. Because our series includes patients treated with anchorless repair, the efficacy of the anchorless transosseous technique is of relevance. Various examinations of the transosseous technique with distal knots have reported good outcomes. Van Riet et al. ${ }^{9}$ reported that 3 of 14 patients experienced rerupture, with 1 experiencing a stitch abscess. An additional study reported that in a series of 10 patients, 1 patient experienced rerupture. ${ }^{2}$ Finally, a 2015 examination of 8 repairs found no reruptures but reported that 1 patient required ulnar nerve release. ${ }^{23}$

A single-row suture anchor technique has also shown good outcomes, with a 2012 case series of 5 repairs showing no rerupture and reporting an average DASH score of 1.4 (range, $0-7 ; \mathrm{SD}, 3$ ) and average MEPS of 95.8 (range, 79-100; SD, 9). ${ }^{24}$ Subsequent studies have aimed to compare distal triceps repair techniques.

Horneff et $\mathrm{al}^{25}$ retrospectively reviewed 56 cases, comparing a transosseous repair with suture anchor constructs, and examined rerupture rates and patient satisfaction, as well as the VAS score, MEPS, and DASH score. They contacted patients by telephone, with an average follow-up period of 4.3 years. They found no statistically significant difference in patient satisfaction, rerupture rate, MEPS, or VAS score based on construct type. They did, however, find a statistically significant difference in the DASH score, with the transosseous group averaging 3 points lower. Although statistically significant, this difference is not believed to be clinically relevant because it has been shown that the minimal clinically important difference for the DASH score is between 10 and 12 points. $^{26}$

Horneff et al. ${ }^{25}$ should be commended for providing a comparative analysis of transosseous repair and suture anchor constructs in their retrospective review. The group size was adequate to ensure power to detect the clinically significant difference of 10 points in the MEPS, ${ }^{27}$ thus showing noninferiority of either technique examined. Nevertheless, this trial was not without limitations, including interviews being conducted by phone, lack of cost analysis, and the grouping of hybrid repairs that used both anchors and sutures into the anchor group. It is possible that the decision to group procedures in this manner serves as a confounder in the comparison between techniques.

Other groups, including Waterman et al., ${ }^{28}$ have performed retrospective analyses of distal triceps ruptures repaired by transosseous bone tunnels and suture anchors for insertional tears. Waterman et al. examined 30 transosseous and 13 anchor repairs and found no reruptures at minimum 1-year follow-up. No differences in complications, VAS scores, MEPS values, or QuickDASH (Quick Disabilities of the Arm Shoulder and Hand) scores were detected. However, pre-existing enthesopathy was determined to be associated with increased complications, including pain, numbness, tendon calcification, subcutaneous adhesions, cyst formation, and wound dehiscence. Although Waterman et al. provided an adequate comparative analysis of patient-reported outcomes in patients with and without pre-existing olecranon enthesopathy, their case series is limited. The lack of a power analysis and the inclusion of cases with various operative indications and surgical techniques suggest that further analyses are required to determine additional risk factors for postoperative complications and to describe clinical outcomes after distal triceps repair.

\section{Limitations}

The limitations of our study include the small sample size and lack of a comparison group; moreover, no biomechanical evaluation of our repair was performed. Strengths include long-term follow-up with validated outcomes, technical simplicity, potential for decreased knot discomfort, and low cost of surgery. Further comparison studies examining clinical outcomes and cost are necessary to determine the ideal repair technique that maximizes clinical outcomes and minimizes cost.

\section{Conclusions}

This case series of triceps tendon repairs using transosseous tunnels and proximally based knots showed favorable postoperative elbow function based on validated outcome measures.

\section{References}

1. Madsen M, Marx RG, Millett PJ, Rodeo SA, Sperling JW, Warren RF. Surgical anatomy of the triceps brachii tendon: Anatomical study and clinical correlation. Am J Sports Med 2006;34:1839-1843.

2. Sierra RJ, Weiss NG, Shrader MW, Steinmann SP. Acute triceps ruptures: Case report and retrospective chart review. J Shoulder Elbow Surg 2006;15:130-134.

3. Stucken C, Ciccotti MG. Distal biceps and triceps injuries in athletes. Sports Med Arthrosc Rev 2014;22:153-163.

4. Sollender JL, Rayan GM, Barden GA. Triceps tendon rupture in weight lifters. J Shoulder Elbow Surg 1998;7: 151-153.

5. Keener JD, Sethi PM. Distal triceps tendon injuries. Hand Clin 2015;31:641-650.

6. Shuttlewood K, Beazley J, Smith CD. Distal triceps injuries (including snapping triceps): A systematic review of the literature. World J Orthop 2017;8:507-513.

7. Marinello PG, Peers S, Sraj S, Evans PJ. A treatment algorithm for the management of distal triceps ruptures. Tech Hand Up Extrem Surg 2015;19:73-80.

8. Balazs GC, Brelin AM, Dworak TC, et al. Outcomes and complications of triceps tendon repair following acute rupture in American military personnel. Injury 2016;47: 2247-2251. 
9. van Riet RP, Morrey BF, Ho E, O’Driscoll SW. Surgical treatment of distal triceps ruptures. J Bone Joint Surg Am 2003;85:1961-1967.

10. Dorweiler MA, Van Dyke RO, Siska RC, Boin MA, DiPaola MJ. A comparative biomechanical analysis of 2 double-row, distal triceps tendon repairs. Orthop J Sports Med 2017;5:2325967117708308.

11. Yeh PC, Stephens KT, Solovyova O, et al. The distal triceps tendon footprint and a biomechanical analysis of 3 repair techniques. Am J Sports Med 2010;38:1025-1033.

12. Ng T, Rush LN, Savoie FH. Arthroscopic distal triceps repair. Arthrosc Tech 2016;5:e941-e945.

13. Sarokhan AK, Leung NL. Acute triceps tendon repair: A technique utilizing 3 curved tunnels and proximal knots. Arthrosc Tech 2019;8:e705-e712.

14. Mirzayan R, Acevedo DC, Sodl JF, et al. Operative management of acute triceps tendon ruptures: Review of 184 cases. Am J Sports Med 2018;46:1451-1458.

15. Blumberg N, Arbel R, Dabby D. Avulsion of the triceps tendon during acceleration stress. Aviat Space Environ Med 2002;73:931-933.

16. Carpenter SR, Stroh DA, Melvani R, Parks BG, Camire LM, Murthi AM. Distal triceps transosseous cruciate versus suture anchor repair using equal constructs: A biomechanical comparison. J Shoulder Elbow Surg 2018;27: 2052-2056.

17. Silverstein P. Smoking and wound healing. Am J Med 1992; $93: 22$ S-24S.

18. Durand F, Berthelot P, Cazorla C, Farizon F, Lucht F. Smoking is a risk factor of organ/space surgical site infection in orthopaedic surgery with implant materials. Int Orthop 2013;37:723-727.

19. Walker CM, Noonan TJ. Distal triceps tendon injuries. Clin Sports Med 2020;39:673-685.
20. Mair SD, Isbell WM, Gill TJ, Schlegel TF, Hawkins RJ. Triceps tendon ruptures in professional football players. Am J Sports Med 2004;32:431-434.

21. Finstein JL, Cohen SB, Dodson CC, et al. Triceps tendon ruptures requiring surgical repair in National Football League players. Orthop J Sports Med 2015;3: 2325967115601021.

22. Dunn JC, Kusnezov N, Fares A, et al. Outcomes of triceps rupture in the US military: Minimum 2-year follow-up. Hand (N Y) 2019;14:197-202.

23. Kose O, Kilicaslan OF, Guler F, Acar B, Yuksel HY. Functional outcomes and complications after surgical repair of triceps tendon rupture. Eur J Orthop Surg Traumatol 2015;25:1131-1139.

24. Bava ED, Barber FA, Lund ER. Clinical outcome after suture anchor repair for complete traumatic rupture of the distal triceps tendon. Arthroscopy 2012;28:1058-1063.

25. Horneff JG III, Aleem A, Nicholson T, et al. Functional outcomes of distal triceps tendon repair comparing transosseous bone tunnels with suture anchor constructs. J Shoulder Elbow Surg 2017;26:2213-2219.

26. Franchignoni F, Vercelli S, Giordano A, Sartorio F, Bravini E, Ferriero G. Minimal clinically important difference of the Disabilities of the Arm, Shoulder and Hand outcome measure (DASH) and its shortened version (QuickDASH). J Orthop Sports Phys Ther 2014;44:30-39.

27. Cusick MC, Bonnaig NS, Azar FM, Mauck BM, Smith RA, Throckmorton TW. Accuracy and reliability of the Mayo Elbow Performance Score. J Hand Surg Am 2014;39: 1146-1150.

28. Waterman BR, Dean RS, Veera S, et al. Surgical repair of distal triceps tendon injuries: Short-term to midterm clinical outcomes and risk factors for perioperative complications. Orthop J Sports Med 2019;7:2325967119839998. 